TABLE II. Transformation between laboratory-fixed (unprimed) and rotating (double primed) coordinate systems.

$$
\begin{aligned}
& X=Z^{\prime \prime} \cos \varphi-Y^{\prime \prime} \sin \varphi \\
& Y=Z^{\prime \prime} \sin \varphi+Y^{\prime \prime} \cos \varphi \\
& Z=X^{\prime \prime}
\end{aligned}
$$

$(\Theta=\pi / 2, \varphi)$ plane, the two theories predict rather different cross sections. The DWBA yields $(d \sigma)$ $d \Omega) \propto\left|J_{1}(q R)\right|^{2}$, while the MO approach leads to a dominant $\left|J_{0}(q R)\right|^{2}$. Experiment seems to suggest that the latter function better represents the data. Other angular-momentum transfers $(\Delta l=0$ and $\Delta l$ $=2 \hbar$ ) do not have this same striking discrepancy between the DWBA and MO approach.

It should be stressed that the approximations used in deriving these results severely limit their generality. It is a sensitive function of energy and particular system considered that allows the angular distribution to have diffractive features while occurring slowly enough compared to internal motion to insure the validity of the MO approximation. In general, a full coupledchannels calculation including a treatment of the relative motion with nuclear and Coulomb distortions and the effect of both core potentials exactly is required. Such calculations are presently under investigation.

Since the conclusions reached here are based primarily on the geometrical character of the
MO perturbation, we expect that for the $\Delta l=1 \hbar$ transitions the MO approach will predict an angular distribution out of phase with that of the DWBA even in more complete calculations. Still, it is interesting to see that nature may have provided a few examples where these approximations are valid, and these analytic results hold.

This work was supported under U. S. Energy Research and Development Administration Contract No. EY-76-C-02-3074.

1J. S. Blair, Phys. Rev. 115, 928 (1959).

${ }^{2}$ A. Dar, Nucl. Phys. 55, 305 (1964).

${ }^{3} \mathrm{~K}$. R. Greider, in Nuclear Reactions Induced by Heavy Ions (North-Holland, Amsterdam, 1970), p. 217.

${ }^{4}$ R. M. DeVries et al., Phys. Rev. Lett. 32, 680 (1974).

${ }^{5}$ P. D. Bond et al., Phys. Rev. Lett. 36, 300 (1976).

${ }^{6}$ T. Motobayashi et al., Phys. Rev, Lett. 36,390 (1976).

${ }^{7}$ D. R. Bates et al., Proc. Ray. Soc., Ser. A 216, 437 (1953).

${ }^{8}$ H. S. W. Massey, E. H. S. Burhop, and H. B. Gilbody, Electronic and Ionic Impact Phenomena (Oxford Univ. Press, Oxford, England, 1974), Vol. IV.

${ }^{9} \mathrm{~K}$. Pruess et al., Phys. Lett. 33B, 197 (1970).

${ }^{10} \mathrm{D}$. Scharnweber et al., Nucl. Phys. A164, 257 (1971).

${ }^{11}$ J. Y. Park et al., Phys. Rev. C 10, 967 (1974).

${ }^{12}$ This localization minimizes difficulties associated with the incorrect asymptotic behavior (spurious rotation) of the molecular-orbital states since an expansion into molecular orbitals is required only within the region of transfer. This feature is particular to the nuclear case compared to atomic physics calculations.

\title{
Photon Antibunching in Resonance Fluorescence
}

\author{
H. J. Kimble, ${ }^{(a)}$ M. Dagenais, and L. Mandel \\ Department of Physics and Astronomy, University of Rochester, Rochester, New York 14627
}

(Received 22 July 1977)

\begin{abstract}
The phenomenon of antibunching of photoelectric counts has been observed in resonance fluorescence experiments in which sodium atoms are continuously excited by a dye-laser beam. It is pointed out that, unlike photoelectric bunching, which can be given a semiclassical interpretation, antibunching is understandable only in terms of a quantized electromagnetic field. The measurement also provides rather direct evidence for an atom undergoing a quantum jump.
\end{abstract}

The tendency of photons in a light beam emitted by a thermal equilibrium source to arrive in bunches, rather than strictly at random, has been well known since the classic experiments of Hanbury Brown and Twiss. ${ }^{1}$ The bunching phenomenon was studied more explicitly in time-resolved correlation experiments, ${ }^{2}$ and it was con- firmed that the joint probability density of photodetection $P_{2}(t, t+\tau)$ by a phototube at two times $t$ and $t+\tau$ is greatest when $\tau$ is near zero, and falls to a constant lower value once $\tau$ appreciably exceeds the coherence time. It is possible to look on the bunching phenomenon as a characteristic quantum feature of thermal bosons. If the wave 
functions of neighboring bosons overlap, when the states are symmetrized the resulting interference increases the probability of detecting one boson near another one.

Nevertheless, it is well known that we may also account for the bunching of photoelectric pulses in terms of the fluctuations of a completely classical field of instantaneous intensity $I(t)$ $\equiv E_{i} *(t) E_{i}(t)$. The joint probability density $P_{2}(t, t$ $+\tau)$ can be shown to be given by ${ }^{3}$

$$
P_{2}(t, t+\tau)=\alpha^{2}\langle I(t) I(t+\tau)\rangle,
$$

where $\alpha$ is a constant characterizing the efficiency of the detector, and the average is to be taken over the ensemble of all realizations of the electromagnetic field $E_{i}(t)$. If we introduce the normalized correlation function $(\Delta I \equiv I-\langle I\rangle)$

$$
\lambda(\tau) \equiv\langle\Delta I(t) \Delta I(t+\tau)\rangle /\langle I(t)\rangle\langle I(t+\tau)\rangle,
$$

we may reexpress Eq. (1) in the form

$$
P_{2}(t, t+\tau)=\alpha^{2}\langle I(t)\rangle\langle I(t+\tau)\rangle[1+\lambda(\tau)] .
$$

Since $\lambda(\tau) \leqslant \lambda(0)$ and $\lambda(\infty)=0$ for an ergodic process, this equation shows at once that the twofold detection probability is greater for time intervals $\tau$ near zero than for long intervals.

In the fully quantized treatment of the same problem given by Glauber, ${ }^{4} \hat{I}(t)$ becomes a Hilbert-space operator ${ }^{5} \hat{E}_{i}{ }^{\dagger}(t) \hat{E}_{i}(t)$, and the correlations have to be written in normal order (::) and in time order $(\tau)$ in the form $\langle\tau: \hat{I}(t) \hat{I}(t+\tau):\rangle$. With this change Eqs. (1) - (3) remain valid. The correlation between the two expressions is provided by the diagonal coherent-state $(|\{v\}\rangle)$ representation of the density operator $\hat{\rho}$ of the free field $d^{6,4}$ :

$$
\hat{\rho}=\int \varphi(\{v\})|\{v\}\rangle\langle\{v\}| d\{v\} .
$$

This allows expectation values of normally or dered operators to be expressed just like $c$-number averages, with the weighting or phase-space functional $\varphi(\{v\})$ playing the formal role of the "probability functional." For a quantum field we then merely replace classical averages like $\langle I(t) I(t+\tau)\rangle_{\text {by }}\langle I(t) I(t+\tau)\rangle_{\varphi}$. However $\varphi(\{v\})$ has the full character of a classical probability functional only for those states of the electromagnetic field for which a classical analog exists [e.g., for thermal fields having Gaussian $\varphi(\{v\})]$, when the quantum and classical descriptions lead to similar conclusions.

On the other hand, there also exist quantum states of the field that have no classical description, for which $\varphi(\{v\})$ may be negative or highly singular and does not have the character of a probability density. For such states $\lambda(0)$ may become negative, and Eq. (3) then predicts the opposite effect, or antibunching near $\tau=0$, and the joint probability density $P_{2}(t, t+\tau)$ may increase with $\tau$ from $\tau=0$. Such antibunching or negative photoelectric correlation is an explicit feature of a quantum field, ${ }^{7-11}$ and its observation would provide rather direct evidence for the existence of optical photons, unlike positive correlation effects that have a semiclassical explanation.

It has recently been predicted that the electromagnetic field radiated by a driven two-level atom in the presence of a continuous exciting field has the correlation function ${ }^{10}$

$$
\langle\tau: \hat{I}(t) \hat{I}(t+\tau):\rangle=\langle\hat{I}(t)\rangle\left\langle\hat{I}_{G}(\tau)\right\rangle,
$$

where $\left\langle\hat{I}_{G}(\tau)\right\rangle$ is the mean light intensity radiated at time $\tau$ following the turn-on of the interaction when the atom starts in the lower (or ground) state. Since $\left\langle\hat{I}_{G}(\tau)\right\rangle$ is necessarily zero for $\tau=0$ and increases with $\tau$ from zero, such a driven atom is an example of a source exhibiting photon antibunching, with $\lambda(0)=-1$ in the steady state. We wish to report the observation of photoelectric antibunching in resonance fluorescence experiments on single atoms.

The principle of the experiment is illustrated in Fig. 1. Atoms of sodium in an atomic beam are excited by the light beam of a tunable dye laser propagating into the plane of the diagram. The light is stabilized both in intensity (to a few percent) and in frequency (to about $1 \mathrm{MHz}$ )。 The laser is tuned to the $\left(3^{2} S_{1 / 2}, F=2\right)$ to $\left(3^{2} P_{3 / 2}, F=3\right)$ transition, and by optical prepumping with circularly polarized light in a weak magnetic field just before the final interaction, the sodium atoms are prepared in the $3^{2} S_{1 / 2}, F=2, m_{F}=2$ state, from which the only allowed transition is to the $3^{2} P_{3 / 2}, F=3, m_{F}=3$ state. $^{12}$ This procedure as-

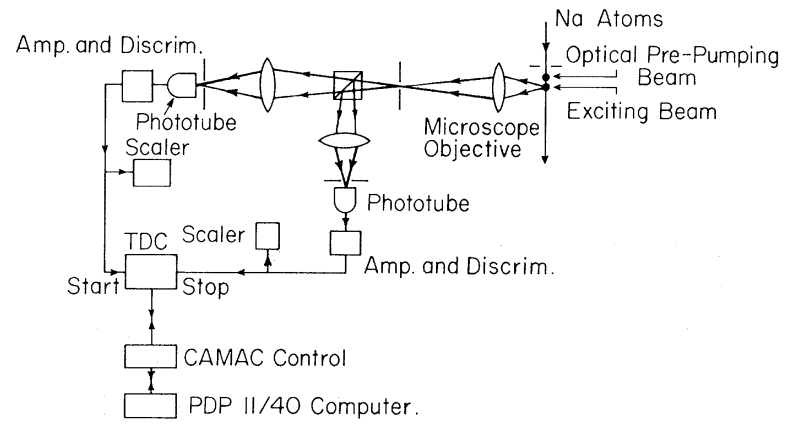

FIG. 1. Outline of the principal elements of the experiment. 


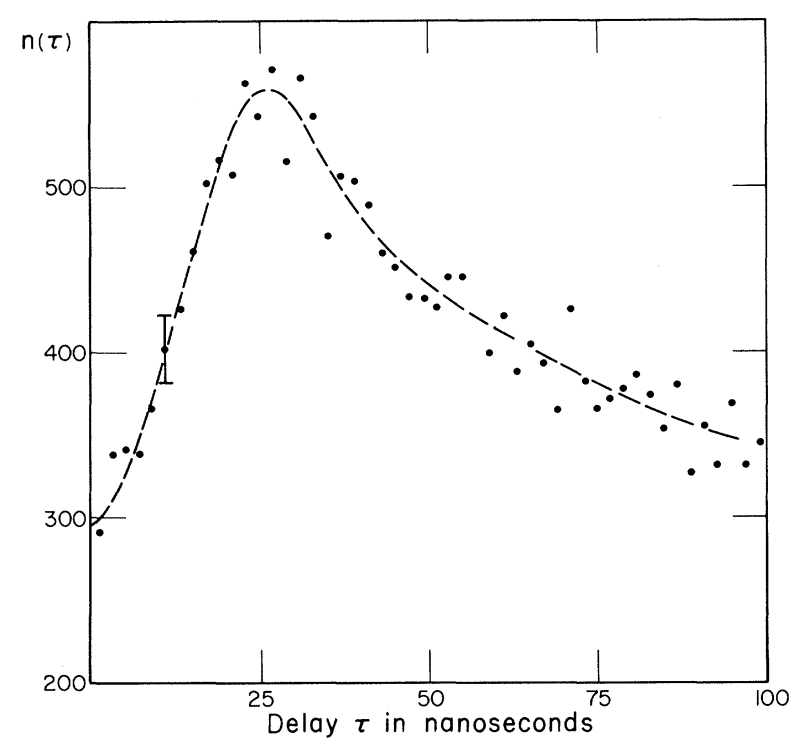

FIG. 2. The number of recorded pulse pairs $n(\tau)$ as a function of the time delay $\tau$ in nanoseconds. The growth of $n(\tau)$ from $\tau=0$ shows antibunching. The bars on one point indicate statistical uncertainties corresponding to one standard deviation. The broken line just outlines the trend.

sures that the atoms behave like two-level quantum systems. The resonant optical field seen by each atom has a power density of about $70 \mathrm{~mW} /$ $\mathrm{cm}^{2}$, corresponding to a Rabi frequency $\Omega$ between 2 and 2.5 times the Einstein $A$ coefficient (in the language of Ref. $10, \Omega / \beta \sim 4-5$ ). The fluorescent light emitted is collected at right angles to both the laser and atomic beams by a microscope objective that images a region of linear dimensions of order $100 \mu \mathrm{m}$ onto an aperture. The light emerging from this aperture is divided into approximately equal parts by a beam splitter, and is then further imaged onto two photomultiplier tubes. The atomic beam also is passed through a $100-\mu \mathrm{m}$ aperture, and the atomic current is held low enough to ensure that no more than one or two atoms in the field are able to contribute to the collected fluorescence radiation at the same time. After amplification and pulse shaping, the pulses from the two detectors are fed to the start and stop inputs of a time-to-digital converter (TDC), where the time intervals $\tau$ between start and stop pulses are digitized in units of $0.5 \mathrm{nsec}$ and stored. The number of events $n(\tau)$ stored at address $\tau$ is therefore a measure of the joint photoelectric detection probability density $P_{2}(t, t+\tau)$. The TDC is under the control of a PDP 11/40 computer, where the information is ultimately recorded.
Figure 2 shows the number of photoelectric pulse pairs $n(\tau)$ recorded for various time delays $\tau$ in intervals of $2 \mathrm{nsec}$, before any corrections are applied to the data. The number $n(\tau)$ is related to the intensity correlation function at the two detectors by

$$
\langle n(\tau)\rangle=N_{s} \Delta \tau \alpha_{2}\left\langle\tau: \hat{I}_{1}(t) \hat{I}_{2}(t+\tau):\right\rangle /\left\langle\hat{I}_{1}(t)\right\rangle,
$$

where $\hat{I}_{1}(t)$ and $\hat{I}_{2}(t)$ are expressed in units of photons per second and suffixes 1 and 2 refer to the start and stop channels, respectively; $N_{s}$ is the number of start pulses received $\left(9 \times 10^{6}\right.$ in this experiment); $\alpha_{2}$ is the detection efficiency in the stop channel; and $\Delta \tau$ is the channel width (2 nsec for the data in Fig. 2). It will be seen that $n(\tau)$ increases with $\tau$ from its smallest value at $\tau=0$, as predicted, so that the experiment shows unmistakable evidence for antibunching of photoelectric pulses.

In order to attempt a more quantitative comparison with the theory we have to make a number of corrections to the data. In the first place there are accidental pair correlations $n(\tau)$ contributed by scattered laser light that enters the two phototubes and provides a background. Although the amount of scattered background light has been held to several times less than the fluorescent light by use of apertures and imaging systems, it still represents a problem in this experiment, because of the requirement to study single atoms so far as possible. In order to correct for this we express the total field at each detector as the sum of two parts $\hat{E}_{i}{ }^{(1)}+e_{i}{ }^{(1)}$ and $\hat{E}_{i}{ }^{(2)}+e_{i}{ }^{(2)}$, where $\hat{E}_{i}$ represents the fluorescent field and $e_{i}$ the scattered laser field that we treat as a $c$-number, and we substitute in Eq. (6). The correlation function then breaks into sixteen distinct terms, most of which have been calculated in Ref. 10. If we discard terms in $e, e^{2}$, and $|e|^{2} e$, on the ground that the scattered laser field probably has a rapidly oscillating phase across the cathode of the photodetector, so that the corresponding interference terms sum almost to zero, we find that

$$
\begin{aligned}
\langle n(\tau)\rangle \approx\left(N_{s} \Delta \tau / \mathbb{R}_{1}\right) & {\left[\mathbb{R}_{1} \mathbb{R}_{2}+R_{1} R_{2} \lambda(\tau)\right.} \\
+ & \left.2\left(R_{1} R_{2} r_{1} r_{2}\right)^{1 / 2}|\gamma(\tau)|\right] .
\end{aligned}
$$

Here $R_{i}, r_{i}$, and $R_{i}(i=1,2)$ are the counting rates at photodetector $i$ contributed by the fluorescent light alone, by the scattered laser light alone, and by both fluorescent and scattered light together. The last two are of course measurable directly with the atomic beam turned off and on, 


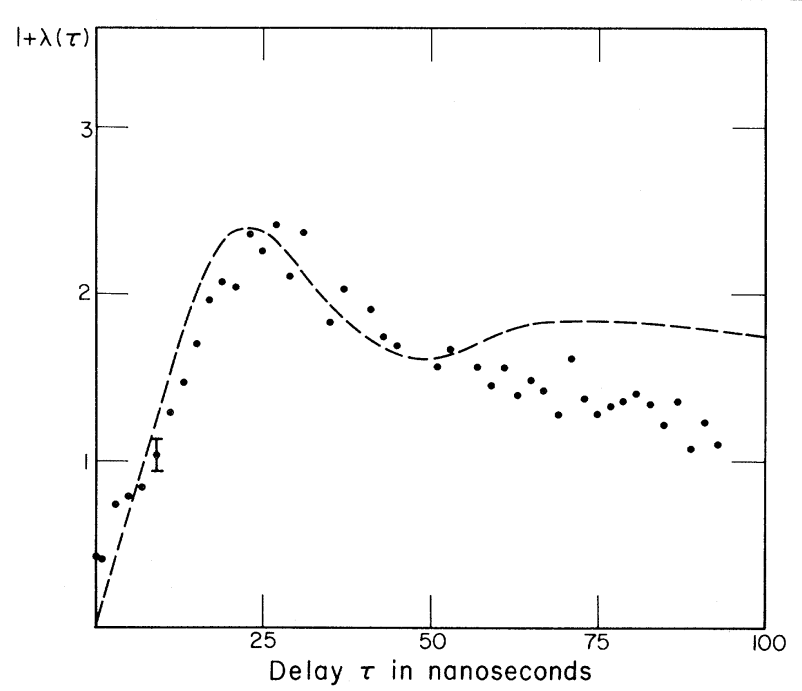

FIG. 3. Values of $1+\lambda(\tau)$ derived from the data. The broken curve shows the theoretically expected form of $\left\langle\hat{I}_{G}(\tau)\right\rangle$ (with $\Omega / \beta=4$ ) for a single atom, arbitrarily normalized to the same peak.

and $R_{i}$ is given by $R_{i}-r_{i}$. The following counting rates were used in the measurement: $R_{1}=13600 /$ sec, $R_{2}=16600 / \mathrm{sec}, r_{1}=2430 / \mathrm{sec}, r_{2}=4450 / \mathrm{sec}$. $\gamma(\tau)$ is the normalized second-order correlation function of the fluorescent field, which was first calculated by Mollow. ${ }^{13}$

The result of applying Eq. (7) to the data is shown in Fig. 3, where we have plotted $1+\lambda(\tau)$ against $\tau$. It will be seen that the initial value $\lambda(0)$ is approximately -0.6 , rather than -1 as predicted theoretically. We believe that this is probably because the detected fluorescence is not always produced by a single atom, but sometimes by two or more. The atomic flux is difficult to measure with accuracy, but from the properties of the detectors and the geometry we estimate the mean number of contributing atoms to be around 1, within a factor of 2 or 3 . For the field produced by two radiating atoms located at random positions the expected value of $\lambda(0)$ would be -0.5 .

As the derived value of $1+\lambda(0)$ is larger than predicted for a single radiating atom, we cannot make really meaningful quantitative comparisons with the single-atom theory. Nevertheless, in order to show that the measured behavior of 1 $+\lambda(\tau)$ has at least the general form predicted theoretically ${ }^{9,10}$ for $\left\langle\hat{I}_{G}(\tau)\right\rangle$, we have also indicated the predicted form of $\left\langle\hat{I}_{G}(\tau)\right\rangle$ in Fig. 3 for a Rabi frequency $\Omega=4 \beta$, normalized rather arbitrarily to the same maximum. The differences between this curve and the derived values of $1+\lambda(\tau)$ for large $\tau$ are partly attributable to the short transit time of atoms moving with average velocity around $10^{5} \mathrm{~cm} / \mathrm{sec}$ through the field of view sampled by the photodetectors. This causes the measured values of $1+\lambda(\tau)$ to be biased downwards for large $\tau$ as compared with those for short $\tau$. Nevertheless, the measured correlation function is at least qualitatively as expected.

Finally, we would like to point out that the evidence provided for photon antibunching near $\tau=0$ is, at the same time, rather direct evidence for an atom undergoing a quantum jump. Although the state of an excited atom evolves continuously in the absence of an observation, the theory predicts a sudden return to the lower state when a photon is detected. That $P_{2}(t, t+\tau)$ vanishes when $\tau=0$ for a single atom may be regarded as a reflection of the fact that the atom, having emitted a photon at time $t$, is unable to radiate again immediately after having made a quantum jump back to the lower state. The quantum nature of the radiation field and the quantum jump in emission, which are of course inextricably connected, are therefore both manifest in these photoelectric correlation measurements.

This work was supported by the National Science Foundation.

(a) Present address: General Motors Research Laboratories, Warren, Mich. 48063.

${ }^{1}$ R. Hanbury Brown and R. Q. Twiss, Nature (London) 177, 27 (1956), and Proc. Roy. Soc. London, Ser. A $\underline{242}, 300$ (1957), and 243, 291 (1957).

${ }^{2}$ B. L. Morg an and L. Mandel, Phys。Rev. Lett. 16 , 1012 (1966); D. B. Scarl, Phys。 Rev. Lett. 17, 663 (1966); D. T. Phillips, H. Kleinman, and S. P. Davis, Phys. Rev. 153, 113 (1967).

${ }^{3}$ E. M。 Purcell, Nature (London) 178, 1449 (1956); L. Mandel, Proc. Phys. Soc., London 72, 1037 (1958), and 74,233 (1959); L。 Mandel, in Progress in Optics, edited by E. Wolf (North-Holland, Amsterdam, 1963), Vol. 2, p. 181; L. Mandel, E. C. G. Sudarshan, and E. Wolf, Proc. Phys. Soc., London 84, 435 (1964).

${ }^{4}$ R. J. Glauber, Phys. Rev. $\underline{130}, 2529$ (1963), and $\underline{131}$, 2766 (1963).

${ }^{5}$ We label all Hilbert-space operators by the caret.

${ }^{6}$ E. C。 G. Sudarshan, Phys. Rev. Lett. 10, 277 (1963); C. L. Mehta and E。C. G. Sudarshan, Phys. Rev. 138, B274 (1965); J. R. Klauder, Phys. Rev. Lett. 16, $\overline{534}$ (1966); J. R. Klauder and E. C. G。 Sudarshan, Fundamentals of Quantum Optics (Benjamin, New York, 1968).

${ }^{7}$ We should emphasize that for a classical field $\lambda(\tau)$ is necessarily nonnegative only for $\tau=0$, as is clear 
from the definition (2). For other values of $\tau, \lambda(\tau)$ may be negative even for a classical field; this is a characteristic feature of light beats.

${ }^{8}$ D. Stoler, Phys. Rev. Lett. 33, 1397 (1974); M. Kozierowski and $\mathrm{R}$. Tanaś, Opt. Commun. 21, 229 (1977).

${ }^{9}$ H。 J。Carmichael and D. F. Walls, J. Phys. B 9 , 1199 (1976).
${ }^{10}$ H. J. Kimble and L. Mandel, Phys. Rev. A $\underline{13}, 2123$ (1976), and 15, 689 (1977).

${ }^{11}$ L. Mandel, in Progress in Optics, edited by E. Wolf (North-Holland, Amsterdam, 1976), Vol. 13, p. 27, and to be published.

${ }^{12}$ J. A. Abate, Opt. Commun。10, 269 (1974).

${ }^{13}$ B. R. Mollow, Phys. Rev。 $\underline{188}, 1969$ (1969).

\title{
Study of the Breakup of Fast $\mathrm{OH}^{-}$Ions Passing through Carbon Foils and Nitrogen Gas
}

\author{
A. Faibis, G. Goldring, and Z. Vager \\ Department of Nuclear Physics, The Weizmann Institute of Science, Rehovot, Israel
} (Received 28 July 1977)

\begin{abstract}
$\mathrm{OH}^{-}$ions were injected into a "Pelletron"-type tandem electrostatic accelerator and dissociated in the high-voltage terminal by passage through gas and foil strippers. The dissociated protons were further accelerated and their energy spectrum was analyzed in a $90^{\circ}$ magnet. The ions stripped in foils exhibit the effects of a Coulomb explosion and a wake field. The stripping in gas appears to be of a different character. An explanation of this difference is proposed, based on the assumption of a strictly sequential removal of electrons from the ions.
\end{abstract}

Charged molecular beams of high velocity have recently been utilized in a number of experiments ${ }^{1-3}$ as a tool for studying the molecular ions involved, as well as various types of interactions of fast charged particles with solids. In all those measurements the molecular ions were passed through a thin carbon foil which strips off the bonding electrons. The ions dissociate and the distribution of the momenta of the break-up products is then investigated experimentally. All measurements to date were carried out for positive ions. We record here for the first time measurements of a similar nature on a negative ion, $\mathrm{OH}^{-}$. In these measurements we also had occasion to study for the first time the breakup of a molecular ion in gas. This appears to be an entirely different process from the more familiar stripping in solid foils.

The experimental arrangement is shown in Fig. 1. The most essential element is a Model 14UD Pelletron accelerator manufactured by National Electrostatics Corporation, the Koffler accelerator recently assembled at the Weizmann Institute. Negative ions emerge from a "direct extraction" source that is fed with hydrogen gas and a small amount of water vapor. The magnet following the source acts as a mass analyzer and is set to select ions of $17 \mathrm{amu}$ which are essentially all $\mathrm{OH}^{-}$ions. The $\mathrm{OH}^{-}$beam is then accelerated to the high-voltage terminal which was kept at $V$ $=+12.00 \mathrm{MV}$. In the terminal the $\mathrm{OH}^{-}$ions are stripped either in carbon foils or in a nitrogen gas stripper, and out of the stripper emerge positive ions: protons and oxygen ions of various charges. These ions are then further accelerated to ground potential and are momentum analyzed in the $90^{\circ}$ magnet. The magnet was set to transmit the proton beam and the magnet current was modulated by a sawtooth current to allow a fast scanning of the momentum or energy distribution of the protons. The proton beam was monitored in a Faraday cup, and the beam intensity as a function of magnetic field was displayed on a Memoscope. For reasons that will become clear later it was necessary to limit the analyzed pro-

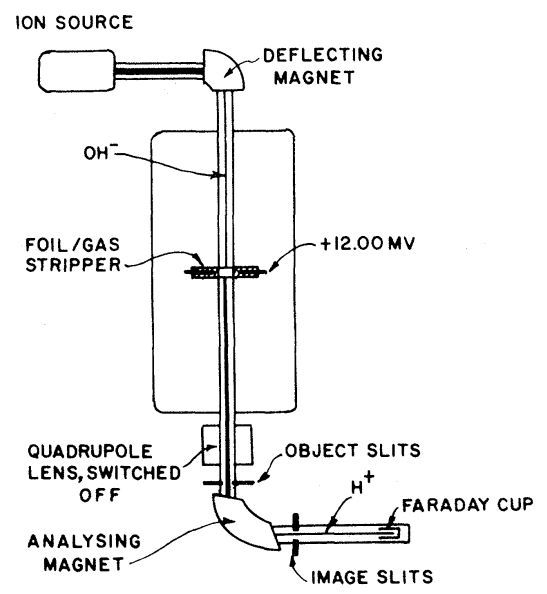

FIG. 1. Schematic drawing of the experimental arrangement. 